\title{
Rationale for strength and skill goals in tongue resistance training: A review
}

\author{
Catriona M. Steele, Gemma L. Bailey, Sonja M. Molfenter \& Erin M. Yeates
}

\section{Authors:}

Catriona M. Steele, Ph.D., CCC-SLP, BRS-S

Scientist, Toronto Rehabilitation Institute

Associate Professor, University of Toronto

Gemma L. Bailey, M.H.Sc., SLP(C)

Speech-Language Pathologist, Toronto Rehabilitation Institute

Sonja M. Molfenter, M.H.Sc., SLP(C)

Speech-Language Pathologist, Toronto Rehabilitation Institute

Erin M. Yeates, M.H.Sc., SLP(C)

Speech-Language Pathologist, Toronto Rehabilitation Institute

\section{Contact Information:}

Catriona M. Steele

550 University Avenue, \#12030,

Toronto, ON, M5G 2A2

Tel: 416-597-3422 X 7603

Email: steele.catriona@torontorehab.on.ca 


\section{Author Biographical Statements:}

Catriona Steele is a scientist and director of the Swallowing Rehabilitation Research Laboratory at the Toronto Rehabilitation Institute. She is an Associate Professor in the Department of Speech-Language Pathology at the University of Toronto and currently serves as Coordinator of ASHA's Special Interest Division 13 (Swallowing and Swallowing Disorders).

Gemma Bailey, Sonja Molfenter and Erin Yeates are speech-language pathologists affiliated with the Swallowing Rehabilitation Research Laboratory at the Toronto Rehabilitation Institute. 


\begin{abstract}
Recent evidence in the dysphagia literature shows that tongue resistance training can be used to improve tongue strength. In this review article, we summarize what is known about the tongue and its role in swallowing. We review the literature on tongue pressure generation capacity (strength) and response to tongue resistance exercises. Tongue skill is introduced as a possible alternate goal in tongue resistance training, based on consideration of related literature regarding neural adaptation and improvements in motor performance resulting from treatment approaches in which goal-oriented practice and performance-specific feedback are provided.
\end{abstract}




\section{Introduction}

The use of oral motor exercises in speech-language pathology has been the subject of much debate (Lof \&Watson, 2008). Clinicians commonly instruct patients in oral range-ofmotion tasks (e.g. tongue lateralization or protrusion) and resistance is sometimes incorporated (e.g., pressing the tongue tip against the cheek wall or a tongue depressor). Critics argue that these exercises are not specific enough to speech or swallowing tasks to yield improvements. Others have pointed out that these exercises probably do not involve sufficient loads to induce changes in the speech or swallowing musculature (Clark, 2003). Amidst this controversy, evidence has been emerging showing that tongue resistance exercise can indeed bring about improvements in tongue strength. In this article, we review what is known about the tongue and its role in swallowing. We review recent literature on tongue pressure generation capacity and response to tongue resistance exercises. Finally, we explore strength and skill as possible goals for tongue resistance training.

\section{What do we know about the tongue and its function in swallowing?}

The oral stage of liquid swallowing unfolds as follows: a) the bolus is taken into the mouth and positioned on the midsagittal groove of the tongue (Chi-Fishman, Stone, \& McCall, 1998); b) The tongue moves up to the palate and pushes forward underneath the bolus (Steele \& van Lieshout, 2009). This forward sliding action initiates posterior movement of the bolus; c) When the bolus reaches the oro-pharyngeal junction, a large downward-backward tongue movement transports the bolus through the pharynx (Steele \& van Lieshout, 2009). Tongue position in the vocal tract is governed by the extrinsic tongue muscles while fine movements and tongue shape are controlled by the intrinsic muscles (Sawczuk \& Mosier, 2001; Takemoto, 
2001). Tongue elevation towards the palate and the application of tongue pressure to the bolus are likely to involve both extrinsic and intrinsic muscle activity.

Previous studies have explored the kinematics of tongue movement during swallowing with different stimuli in healthy individuals (Steele \& van Lieshout, 2004, 2005). Both agerelated changes and stimulus-related differences in tongue movement appear to be restricted to variations in movement duration (Steele \& van Lieshout, 2004; 2009). Longer movement durations are observed in older adults. Longer movement durations were also seen in all participants in anterior tongue dorsum movement for honey-thick juice versus thin liquid stimuli.

\section{What do we know about tongue pressure generation in swallowing?}

Tongue-palate pressures can be measured using air-filled bulbs placed between the tongue and the palate. Several forms of orolingual manometry equipment are available or in development (e.g. Hewitt, et al., 2008; Kieser, et al., 2008; Ono, Hori, \& Nokubi, 2004; Utanohara, et al., 2008). Models vary in the size and number of pressure sensors used, and whether sensor placement is fixed. The Iowa Oral Performance Instrument (IOPI) and the KayPentax Swallowing Signals Lab Orolingual Manometry module are currently the most commonly used. The IOPI uses a single bulb, approximately $2 \mathrm{~cm} \times 1 \mathrm{~cm} \times 0.5 \mathrm{~cm}$, which is held in the mouth behind the alveolar ridge, and connects to a device that displays pressure in kilopascals (Youmans \& Stierwalt, 2006). The KayPentax equipment uses a strip of soft plastic housing 2 or 3 pressure sensors (each approximately $5 \mathrm{~mm}$ in diameter and height), secured to the participant's palate using adhesive. Time-linked waveform data are displayed for each sensor on a screen. The IOPI is ideal as a biofeedback device in therapy; the KayPentax equipment is more commonly used for assessment, treatment baseline and endpoints. 
Preliminary data show that healthy young adults typically generate maximum isometric tongue pressures (MIPs) of $80 \mathrm{kPa}$ (600 mm Hg) (Crow \& Ship, 1996). Nicosia and colleagues (2000) studied healthy elderly individuals (mean age: 81), who had significantly lower MIPs than middle-aged controls (mean age: 51). The reported MIP norms for seniors are $40 \mathrm{kPa}$ (300 mm Hg). Importantly, however, swallowing pressures are submaximal and fall well below the values registered in MIP tasks. Healthy seniors do not show a reduction in swallowing pressures although their upper limit on MIPs is reduced; this is interpreted to reflect reduced functional reserve (Robbins, Levine, Wood, Roecker, \& Luschei, 1995). Swallowing pressures in healthy seniors are, however, reported to be more variable than those in younger adults (Nicosia, et al., 2000).

Limited information is available regarding variations in tongue pressure during swallowing with different stimuli. Significantly higher pressures have been reported for mashed potato versus thin liquids (Pouderoux \& Kahrilas, 1995). Youmans and Stierwalt (2006) reported higher tongue pressures during honey-thick liquid swallows compared to thin liquids. Tongue pressure amplitudes increase during effortful (vs. non-effortful) saliva swallows; when an effortful swallow is performed with intentional emphasis on tongue-palate pressure, greater pharyngeal pressures result (Huckabee \& Steele, 2006).

\section{The importance of tongue strength for swallowing in healthy aging}

Swallowing liquids requires precise control of oral bolus flow; this is achieved by the application of tongue pressure to the bolus. Strength is one component required for tongue pressure application. The literature contains many studies showing that age-related changes in swallowing occur in healthy individuals, distinct from changes that might be expected as a function of disease (e.g., Ekberg \& Feinberg, 1991; Logemann et al., 2000; Rademaker, 
Pauloski, Colangelo, \& Logemann, 1998). These age-related changes are attributed primarily to changes in muscle mass, particularly in the tongue. Additionally, elderly subjects are described to use multiple tongue gestures to reach peak pressure (Nicosia, et al., 2000). Multiple gestures are also typical of hyoid movement in seniors, and are interpreted to reflect age-related atrophy in the tongue and in the pharyngeal and laryngeal musculature (Sheth \& Diner, 1988). Agerelated changes in swallowing physiology do not necessarily imply a concomitant impairment of swallowing however, they introduce risk factors that bring the system closer to the limits of its functional capacity (Logemann, 1990).

Encouragingly, recent research has demonstrated that resistance exercises for the tongue yield improvements in MIP (Robbins, et al., 2005). This was first demonstrated in healthy older adults who performed 90 effortful anterior tongue presses (in 3 sets of 30) with the IOPI on three non-consecutive days of the week over an 8-week period. Exercise loads were set between $60 \%$ and $80 \%$ of each participant's MIP, and were adjusted weekly throughout the protocol. On this regimen, participants demonstrated statistically significant improvements in MIPs, in the order of 7\% compared to baseline, with post-treatment values reaching approximately $50 \mathrm{kPa}$ in magnitude. Robbins and colleagues went on to study this same phenomenon in individuals with dysphagia post stroke (Robbins, et al., 2007). Participants completed 30 anterior and 30 posterior tongue presses (in 3 sets of 10 anterior and 10 posterior), again on three non-consecutive days of the week. Exercise loads were again customized to each participant, between 60 and $80 \%$ of MIP. After 8 weeks of treatment, MIPs had improved to $146 \%$ and $181 \%$ of baseline levels in the anterior and posterior bulb positions, respectively. Fascinatingly, these patients also showed improvements in penetration-aspiration scale scores. Bolus control measures were not reported. Thus, the relationship between tongue strength and bolus control remains in question. 


\section{The possible role of tongue pressure generation skill in swallowing:}

Within the motor control literature, skilled movements are defined as tasks that require the "modification and organization of muscle synergies into effective movement sequences" (Monfils, Plautz \& Kleim, 2005). It seems reasonable to propose that the application of tongue pressure to control a liquid bolus in the mouth is a skilled movement task. Skill components within this task might include the ability to modulate the precise amplitude of pressure applied as well as the timing of pressure generation. At the Toronto Rehabilitation Institute, we have been studying tongue pressure skill and exploring the possibility that versatility in tongue motor function is critical for controlling the flow of liquid stimuli in the oral phase of swallowing. To date, there is no consensus about how tongue pressure skill should be measured. We measure skill as absolute tongue pressure accuracy, that is, distance (either above or below) from a pressure target in $\mathrm{kPa}$, divided by MIP.

We are exploring the idea that a skilled exercise program (focusing on accurate tongue pressure generation) may be used as an effective means of improving swallowing function in individuals with dysphagia secondary to age-related decline, stroke, or other neurological impairment (Yeates, Molfenter, \& Steele, 2008). Previous studies have shown that the repetition and relearning of goal-oriented tasks yields superior long-term functional gains to strength training (Winstein, et al., 2004). In simple terms, we require our patients not only to use their tongue muscles and tongue pressure capacity, but to improve this capacity both in terms of strength and accuracy (Robbins, et al., 2008). While strength training has an important role in the rehabilitation process, motor re-learning may also be important for recovery. Long-term relatively permanent changes in behavior can be expected when appropriate feedback and deliberate task-specific practice are provided. Neural adaptation is thought to lead to gains in 
motor performance beyond those due to strength-associated adaptation of muscle (Connelly, Carnahan, \& Vandervoort, 2000). Cortical motor map changes have been described in response to training-related improvements in synaptic efficiency and motor evoked potential thresholds (Kleim, Barbay, \& Nudo, 1998; Svensson, Romaniello, Arendt-Nielsen, \& Sessle, 2003). We are optimistic that a skill-oriented tongue resistance training protocol might similarly yield durable changes in tongue pressure generation capacity, with functional carryover to swallowing. In summary, the dysphagia literature currently contains strong evidence that resistance training improves tongue strength. Swallowing is, however, a submaximal task, and it remains unclear whether improvements in strength transfer to swallowing function. Tongue pressure skill is a new concept, and still under early investigation. However, it appears that strength gains can be expected as an outcome of treatment that focuses on the generation of precise and accurate tongue pressures (Yeates, et al., 2008). Future studies will need to seek evidence of physiological and functional changes in swallowing function from tongue resistance training protocols, so that we can better understand how to tailor treatment to patient deficits. 


\section{References:}

Chi-Fishman, G., Stone, M., \& McCall, G. N. (1998). Lingual action in normal sequential swallowing. Journal of Speech, Language Hearing Research 41(4), 771-785.

Clark, H. M. (2003). Neuromuscular treatments for speech and swallowing: a tutorial. American Journal of Speech-Language Pathology, 12(4), 400-415.

Connelly, D., Carnahan, H., \& Vandervoort, T. (2000). Practice effects on concentric-eccentric patterns during isokinetic strength training in old adults. Experimental Aging Research, 26, 209-228.

Crow, H. C., \& Ship, J. A. (1996). Tongue strength and endurance in different aged individuals. Journals of Gerontology Series A - Biological Sciences and Medical Sciences, 51(5), M247-M250.

Ekberg, O., \& Feinberg, M. J. (1991). Altered swallowing function in elderly patients without dysphagia: radiologic findings in 56 cases. AJR American Journal of Roentgenology, 156(6), 1181-1184.

Hewitt, A., Hind, J., Kays, S., Nicosia, M., Doyle, J., Tompkins, W., et al. (2008). Standardized instrument for lingual pressure measurement. Dysphagia, 23(1), 16-25.

Huckabee, M. L., \& Steele, C. M. (2006). An analysis of lingual contribution to submental sEMG measures and pharyngeal biomechanics during Effortful Swallow. Archives of Physical Medicine and Rehabilitation, 87(August), 1067-1072.

Pouderoux, P. \& Kahrilas, P. J. (1995). Deglutitive tongue force modulation by volition, volume, and viscosity in humans. Gastroenterology, 108(5), 1418-1426.

Kieser, J. A., Singh, B., Swain, M. V., Ichim, I., Waddell, J. N., Kennedy, D., et al. (2008). Measuring intra-oral pressure: Adaptation of a dental appliance allows measurement during function. Dysphagia, 23, 237-243.

Kleim, J. A., Barbay, S., \& Nudo, R. J. (1998). Functional reorganization of the rat motor cortex following motor skill learning. Journal of Neurophysiology, 80, 3321-3325.

Lof, G. L., Watson, M. M. (2008). A nationwide survey of nonspeech oral motor exercise use: implications for evidence-based practice. Language, Speech \& Hearing Services in the Schools, 39(3), 392-407.

Logemann, J. A. (1990). Effects of aging on the swallowing mechanism. Otolaryngologic Clinics of North America, 23(6), 1045-1056.

Logemann, J. A., Pauloski, B. R., Rademaker, A. W., Colangelo, L. A., Kahrilas, P. J., \& Smith, C. H. (2000). Temporal and biomechanical characteristics of oropharyngeal swallow in younger and older men. Journal of Speech, Language Hearing Research, 43(5), 12641274.

Monfils, M. H., Plautz, E. J., Kleim, J. A. (2005). In search of the motor engram: Motor map plasticity as a mechanism for encoding motor experience. Neuroscientist, 11(5), 471-483.

Nicosia, M. A., Hind, J. A., Roecker, E. B., Carnes, M., Doyle, J., Dengel, G. A., et al. (2000). Age effects on the temporal evolution of isometric and swallowing pressure. Journals of Gerontology Series A-Biological Sciences \& Medical Sciences, 55(11), M634-640.

Ono, T., Hori, K., \& Nokubi, T. (2004). Pattern of tongue pressure on hard palate during swallowing. Dysphagia, 19(4), 259-264.

Rademaker, A. W., Pauloski, B. R., Colangelo, L. A., \& Logemann, J. A. (1998). Age and volume effects on liquid swallowing function in normal women Journal of Speech, Language Hearing Research, 41(2), 275-284. 
Robbins, J., Butler, S. G., Daniels, S. K., Diez Gross, R., Langmore, S., Lazarus, C. L., et al. (2008). Swallowing and dysphagia rehabilitation: translating principles of neural plasticity into clinically oriented evidence. Journal of Speech, Language Hearing Research, 51(1), S276-300.

Robbins, J., Gangnon, R. E., Theis, S. M., Kays, S. A., Hewitt, A. L., \& Hind, J. A. (2005). The effects of lingual exercise on swallowing in older adults. Journal of the American Geriatric Society, 53(9), 1483-1489.

Robbins, J., Kays, S. A., Gangnon, R. E., Hind, J. A., Hewitt, A. L., Gentry, L. R., et al. (2007). The effects of lingual exercise in stroke patients with dysphagia. Archives of Physical Medicine and Rehabilitation, 88(2), 150-158.

Robbins, J., Levine, R., Wood, J., Roecker, E. B., \& Luschei, E. (1995). Age effects on lingual pressure generation as a risk factor for dysphagia. Journals of Gerontology Series ABiological Sciences \& Medical Sciences, 50(5), M257-M262.

Sawczuk, A., \& Mosier, K. M. (2001). Neural control of tongue movement with respect to respiration and swallowing. Critical Reviews in Oral Biology and Medicine, 12(1), 18-37.

Sheth, N., \& Diner, W. C. (1988). Swallowing problems in the elderly. Dysphagia, 2(4), 209215.

Steele, C. M., \& van Lieshout, P. H. (2004). Influence of bolus consistency on lingual behaviors in sequential swallowing. Dysphagia, 19(3), 192-206.

Steele, C. M., \& van Lieshout, P. H. (2005). Does barium influence tongue behaviors during swallowing? American Journal of Speech Language Pathology, 14(1), 27-39.

Steele, C. M., \& van Lieshout, P. H. (2009, in press). Tongue movements during water swallowing in healthy young and older adults. Journal of Speech, Language Hearing Research

Svensson, P., Romaniello, A., Arendt-Nielsen, L., \& Sessle, B. J. (2003). Plasticity in corticomotor control of the human tongue musculature induced by tongue-task training. Experimental Brain Research, 152(1), 42-51.

Takemoto, H. (2001). Morphological analyses of the human tongue musculature for threedimensional modeling. Journal of Speech, Language Hearing Research, 44(1), 95-107.

Utanohara, Y., Hayashi, R., Yoshikawa, M., Yoshida, M., Tsuga, K., Akagawa, Y., et al. (2008). Standard values of maximum tongue pressure taken using newly developed disposable tongue pressure measurement device. Dysphagia, 23(3), 286-290.

Winstein, C. J., Rose, D. K., Tan, S. M., Lewthwaite, R., Chui, H. C., \& Azen, S. P. (2004). A randomized controlled comparison of upper-extremity rehabilitation strategies in acute stroke: A pilot study of immediate and long-term outcomes. Archives of Physical Medicine and Rehabilitation, 85, 620-628.

Yeates, E. M., Molfenter, S. M., \& Steele, C. M. (2008). Improvements in tongue strength and pressure-generation precision following a tongue-pressure training protocol in older individuals with dysphagia: Three case reports. Clinical Interventions in Aging, 3(4), 735-747.

Youmans, S. R., \& Stierwalt, J. A. (2006). Measures of tongue function related to normal swallowing. Dysphagia, 21(2), 102-111. 


\section{Proposed CEU Questions:}

1. Which of the following are reported outcomes of tongue resistance training?
a. Improved maximum isometric tongue pressures
b. Reduced maximum isometric tongue pressures
c. Decreased hyolaryngeal excursion
d. Increased vallecular residue
e. Decreased intrabolus pressure

2. Which of the following criticisms have been made regarding oral motor exercises?

a. These exercises are effective for improving speech but not swallowing function

b. These exercises do not involve loads sufficient to induce muscle changes

c. These exercises are effective for improving swallowing but not speech

d. These exercises induce excessive fatigue in the orofacial musculature

e. These exercises improve endurance but do not change strength of the orofacial musculature

3. Which of the following are true about tongue strength in healthy adults?

a. Older adults experience an age-related increase in maximum isometric tongue pressure values

b. Older adults do not experience age-related reductions in maximum isometric tongue pressure values

c. Older adults do not experience age-related reductions in swallowing pressures

d. Older adults experience an age-related decrease in swallowing pressures

e. Older adults experience an age-related increase in swallowing pressures

4. Which of the following patterns describes the direction and sequence of tongue movement in the oral phase of swallowing?

a. upward and backward, then downward

b. upward and forward, then downward and backward

c. backward then downward

d. downward and forward, then upward and backward

e. downward and backward, then forward

5. Which of the following might reflect tongue pressure skill?

a. The ability to form a midsagittal groove in the tongue

b. The ability to elevate the tongue towards the palate

c. The ability to generate maximum isometric pressures of $40 \mathrm{kPa}$ or greater

d. The ability to vary the amplitude of pressure applied to a bolus

e. The ability to generate swallowing pressures of $40 \mathrm{kPa}$ or greater 